Annals of Pure and Applied Mathematics

Vol. 16, No. 1, 2018, 223-234

ISSN: 2279-087X (P), 2279-0888(online)

Published on 16 January 2018

www.researchmathsci.org

DOI: http://dx.doi.org/10.22457/apam.v16n1a24

Annals of

Pure and Applied

Mathematics

\title{
A Study on Domination Sets in Vague Graphs with
} Novel Applications

\author{
Hossein Rashmanlou ${ }^{1}$, Abolfazl Lakdashti ${ }^{2}$ and Shabanali Safari Sabet ${ }^{3}$ \\ ${ }^{1,2}$ Department of Computer Science \\ University College of Rouzbahan, Sari, Iran \\ ${ }^{3}$ Department of Mathematics, Islamic Azad University \\ Central Tehran Branch, Tehran, Iran \\ Email: rashmanlou.1987@gmail.com; lakdashti@ rouzbahan.ac.ir; \\ ${ }^{3}$ Corresponding author. email: drsafarisabet@gmail.com
}

Received 15 August 2017; accepted 15 December 2017

\begin{abstract}
Abstarct. Theoretical concepts of graphs are highly utilized by computer science applications. Especially in research areas of computer science such as data mining, image segmentation, clustering, image capturing and networking. Fuzzy graph theory has numerous applications in modern sciences and technology such as neural networks, artificial intelligence and decision making. A vague graph is a generalized structure of a fuzzy graph that gives more precision, flexibility, and compatibility to a system when compared with systems that are designed using fuzzy graphs. In this paper, we study some results on point set dominating set and its number of vague graphs and semi complete vague graphs. Likewise, we introduce the bounds with other domination parameters of vague graphs. Finally, we give some applications of point set dominating sets in the other sciences.
\end{abstract}

Keywords: Vague graph, point set domination set, semi complete vague graph

AMS Mathematics Subject Classification (2010): 05C99, 05 C22

\section{Introduction}

Graph theory is an extremely useful tool in solving combinatorial problems in different areas including geometry, algebra, number theory, topology, operations research, biology and social systems. Fuzzy graph theory is finding an increasing number of application in modeling real time systems where the level of information inherent in the system varies with different levels of precision. In 1965, Zadeh [19] first proposed the theory of fuzzy sets. Gau and Buehrer [3] proposed the concept of vague set in 1993, by replacing the value of an element in a set with a subinterval of [0,1]. Namely, a true-membership function $t_{v}(x)$ and a false membership function $f_{v}(x)$ are used to describe the bounderies of the membership degree. The first definition of fuzzy graphs was proposed by Kafmann [4] in 1993, from Zadeh's fuzzy relations [19]. Ramakrishna [7] introduced the concept of vague graphs and studied some of their properties. Akram et al. [1] defined vague hypergraphs. Somasundaram and Somasundaram [17] investigated domination in fuzzy graphs. 
H.Rashmanlou, A.Lakdashti and S.S.Sabet

Domination in intuitionistic fuzzy graphs introduced by Parvathi and Thamizhendhi [6]. Gani et al. [5] studied new concepts of domination in intuitionistic fuzzy graphs. Borzooei et al. [2] defined domination in vague graphs. Rashmanlou et al. [8,9,10,11,12,13,14,15, 16] invetigated some basic results on bipolar fuzzy graphs and vague graphs. In this paper, we study some results on point set dominating set and its number of vague graphs and semi complete vague graph. Also, we introduce the bounds with other domination parameters of vague graphs. Lots of works have been done on fuzzy and related graphs by several researchers [20-43].

\section{Preliminaries}

In this section, we review briefly some definitions in vague graphs and introduce some new notations. A graph is an ordered pair $G=(V, E)$, where $V$ is the set of vertices of $G$ and $E$ is the set of edges of $G$. A fuzzy graph $G=(\sigma, \mu)$ is a pair of functions $\sigma: V \rightarrow[0,1]$ and $\mu: V \times V \rightarrow[0,1]$ with $\mu(u, v) \leq \sigma(u) \wedge \sigma(v)$, for all $u, v \in V$, where $V$ is a finite non-empty set and $\wedge$ denote minimum.

A vague set $A$ in an ordinary finite non-empty set $X$, is a pair $\left(t_{A}, f_{A}\right)$, where $t_{A}: X \rightarrow[0,1], f_{A}: X \rightarrow[0,1]$ are true and false membership functions, respectively such that $0 \leq t_{A}(x)+f_{A}(x) \leq 1$, for all $x \in X$. Note that $t_{A}(x)$ is considered as the lower bound for degree of membership of $x$ in $A$ and $f_{A}(x)$ is the lower bound for negative of membership of $x$ in $A$. So, the degree of membership of $x$ in the vague set $A$, is characterized by the interval $\left[t_{A}(x), 1-f_{A}(x)\right]$. Hence, a vague set is a special case of interval-valued sets studied by many mathematicians and applied in many branches of mathematics.

It is worth to mention here that interval valued fuzzy sets are not vague sets. In interval-valued fuzzy sets, an interval valued membership value is assigned to each element of the universe considering the evidence for $x$ only, without considering evidence against $x$. In vague sets both are independently proposed by the decision maker. This makes a major difference in the judgment about the grade of membership. A vague relation is a generalization of a fuzzy relation. Let $X$ and $Y$ be ordinary finite non-empty sets. We call a vague relation to be a vague subset of $X \times Y$, that is an expression $R$ defined by

$$
R=\left\{\left\langle(x, y), t_{R}(x, y), f_{R}(x, y)\right\rangle \mid x \in X, y \in Y\right\}
$$

where $t_{R}: X \times Y \rightarrow[0,1], f_{R}: X \times Y \rightarrow[0,1]$, which satisfies the condition $0 \leq t_{R}(x, y)+f_{R}(x, y) \leq 1$, for all $(x, y) \in X \times Y$. (See [7])

Definition 2.1. [7] $A$ vague graph is defined to be a pair $G=(A, B)$, where $A=\left(t_{A}, f_{A}\right)$ is a vague set on $V$ and $B=\left(t_{B}, f_{B}\right)$ is a vague set on $E \subseteq V \times V$ such that $t_{B}(x y) \leq \min \left(t_{A}(x), t_{A}(y)\right)$ and $f_{B}(x y) \geq \max \left(f_{A}(x), f_{A}(y)\right)$, for each edge $x y \in E$. A vague graph $G$ is said to be connected if there is a path between any two vertices of $G$ The underlying crisp graph of a vague graph $G=(A, B)$, is the graph $G^{*}=(V, E)$ 
A Study on Domination sets in Vague Graphs with Novel Applications

where $V=\left\{v: t_{A}(v)>0\right.$ and $\left.f_{A}(v)>0\right\}$ and $E=\left\{u v: t_{B}(u v)>0, f_{B}(u v)>0\right\} . V$ is called the vertex set and $E$ is called the edge set. A vague graph may be also denoted as $G=(V, E)$.

A vague graph $G$ is said to be strong if $t_{B}\left(v_{i} v_{j}\right)=\min \left\{t_{A}\left(v_{i}\right), t_{A}\left(v_{j}\right)\right\}$ and $f_{B}\left(v_{i} v_{j}\right)=\max \left\{f_{A}\left(v_{i}\right), f_{A}\left(v_{j}\right)\right\}$, for every edge $v_{i} v_{j} \in E$. A vague graph $G$ is said to be complete if $t_{B}\left(v_{i} v_{j}\right)=\min \left\{t_{A}\left(v_{i}\right), t_{A}\left(v_{j}\right)\right\}$ and $f_{B}\left(v_{i} v_{j}\right)=\max \left\{f_{A}\left(v_{i}\right), f_{A}\left(v_{j}\right)\right\}$, for all $v_{i}, v_{j} \in V$.

A vague graph $G$ is said to be $n$-partite (multipartite) if the vertex set $V$ can be partitioned into $n$ subsets $V_{1}, V_{2}, \cdots, V_{n}$ such that: (i) For every $v_{i}, v_{j} \in V_{i}$ and $1 \leq i, j \leq n$, we have

$$
t_{B}\left(v_{i} v_{j}\right)=0 \text { and } f_{B}\left(v_{i} v_{j}\right)=0 \text {. }
$$

(ii) For every $v_{i} \in V_{i}, v_{j} \in V_{j}$ and $1 \leq i, j \leq n$, we have

$$
t_{B}\left(v_{i} v_{j}\right)=0, f_{B}\left(v_{i} v_{j}\right)>0 \text { or } t_{B}\left(v_{i} v_{j}\right)>0, f_{B}\left(v_{i} v_{j}\right)=0 .
$$

Definition 2.2. [2] Let $G=(A, B)$ be a vague graph.

(i) The cardinality of $G$ is defined to be

$$
|G|=\left|\sum_{v_{i} \in V} \frac{1+t_{A}\left(v_{i}\right)+f_{A}\left(v_{i}\right)}{2}+\sum_{v_{i} v_{j} \in E} \frac{1+t_{B}\left(v_{i} v_{j}\right)-f_{B}\left(v_{i} v_{j}\right)}{2}\right| .
$$

(ii) The vertex cardinality of $G$ is defined by

$$
|V|=\sum_{v_{i} \in V} \frac{1+t_{A}\left(v_{i}\right)-f_{A}\left(v_{i}\right)}{2}, \text { for all } v_{i} \in V
$$

(iii) The edge cardinality of $G$ is defined by

$$
|E|=\sum_{v_{i} v_{j} \in E}\left(\frac{1+t_{B}\left(v_{i} v_{j}\right)-f_{B}\left(v_{i} v_{j}\right)}{2}\right), \text { for all } v_{i} v_{j} \in E .
$$

(iv) The number of vertices (the cardinality of $V$ ) is called the order of a vague graph and is denoted by

$$
O(G)=\sum_{v_{i} \in V}\left(\frac{1+t_{A}\left(v_{i}\right)-f_{A}\left(v_{i}\right)}{2}\right), \text { for all } v_{i} \in V .
$$

(v) The number of edges (the cardinality of $E$ ) is called the size of a vague graph and is denoted by

$$
S(G)=\sum_{v_{i} v_{j} \in E}\left(\frac{1+t_{B}\left(v_{i} v_{j}\right)-f_{B}\left(v_{i} v_{j}\right)}{2}\right), \text { for all } v_{i} v_{j} \in E .
$$

The complement of $G=(A, B)$ is a vague graph $\bar{G}=(\bar{A}, \bar{B})$ where $\bar{A}=A$, 
H.Rashmanlou, A.Lakdashti and S.S.Sabet

$\overline{t_{B}}\left(v_{i} v_{j}\right)=\min \left(t_{A}\left(v_{i}\right), t_{A}\left(v_{j}\right)\right)-t_{B}\left(v_{i} v_{j}\right)$ and
$\overline{f_{B}}\left(v_{i} v_{j}\right)=\max \left(f_{A}\left(v_{i}\right), f_{A}\left(v_{j}\right)\right)-f_{B}\left(v_{i} v_{j}\right)$.

Definition 2.3. [2] An edge $e=u v$ of a vague graph is called an effective edge if $t_{B}(u v)=\min \left\{t_{A}(u), t_{A}(v)\right\}$ and $f_{B}(u v)=\max \left\{f_{A}(u), f_{A}(v)\right\}$.

Definition 2.4. [2] Let $G=(V, E)$ be a vague graph.

(i) The neighbourhood of any vertex $v$ is defined as $N(v)=\left(N_{t}(v), N_{f}(v)\right)$, where $N_{t}(v)=\left\{w \in V ; t_{B}(v w)=t_{A}(v) \wedge t_{A}(w)\right\}$ and $N_{f}(v)=\left\{w \in V ; f_{B}(v w)=f_{A}(v) \vee f_{A}(w)\right\} . N[v]=N(v) \cup\{v\}$ is called the closed neighbourhood of $v$.

(ii) The neighbourhood degree of a vertex is defined as $d_{N}(v)=\left(d_{N_{t}}(v), d_{N_{f}}(v)\right)$,

where $d_{N_{t}}(v)=\sum_{w \in N(v)} t_{A}(w)$ and $d_{N_{f}}(v)=\sum_{w \in N(v)} f_{A}(w)$. The minimum

neighbourhood degree is defined as $\delta_{N}(G)=\left(\delta_{N_{t}}(v), \delta_{N_{f}}(v)\right)$, where

$\delta_{N_{t}}(v)=\wedge\left\{d_{N_{t}}(v): v \in V\right\}$ and $\delta_{N_{f}}(v)=\wedge\left\{d_{N_{f}}(v): v \in V\right\}$.

(iii) The effective degree of a vertex $v$ is defined to be sum of the effective edges incident at $v$, and denoted dy $d_{E}(v)$. The minimum effective degree of $G$ is $\delta_{E}(G)=\wedge\left\{d_{E}(v) \mid v \in V\right\}$.

Definition 2.5. [2] Let $G=(V, E)$ be a vague graph and $u, v \in V$.

(i) We say that $u$ dominated $v$ in $G$ if there exist a strong arc between them. A subset $D \subseteq V$ is said to be dominating set in $G$ if for every $v \in V-D$, there exist $u$ in $D$ such that $u$ dominated $v$. The minimum scalar cardinality taken over all dominating set is called domination number and is denoted by $\gamma(G)$. The maximum scalar cardinality of a minimal domination set is called upper domination number and is denoted by the symbol $\Gamma(G)$.

(ii) A subset $D \subseteq V$ is said to be total dominating set in $G$ if every vertex in $V$ is dominated by a node in $D$. The minimum cardinality of all total dominating set is called total domination number and denoted by $\gamma_{t}(G)$.

(iii) An independent set of a vague graph $G$ is a subset $S$ of $V$ such that no two vertices of $S$ are adjacent in $G$.

Definition 2.6. [2] A bipartite vague graph $G$ is said to be complete bipartite vague graph if $t_{B}\left(v_{i} v_{j}\right)=t_{A}\left(v_{i}\right) \wedge t_{A}\left(v_{j}\right)$ and $f_{B}\left(v_{i} v_{j}\right)=f_{A}\left(v_{i}\right) \vee f_{A}\left(v_{j}\right)$, for all $v_{i} \in V_{1}$ and $v_{j} \in V_{2}$. It is denoted by $K_{v_{1 i}, v_{2 i}}$. 
A Study on Domination sets in Vague Graphs with Novel Applications

Definition 2.7. [2] Let $G=(V, E)$ be a connected vague graph with effective edges.

Then $G$ is said to be semi complete vague graph if every pair of vertices have common neighbor in $G$. A vague graph $G$ is said to be purely semi-complete vague graph if and only if $G$ is semi complete vague graph but not complete vague graph.

\section{Point set domination in the vague graphs}

Domination in graphs has many applications to several fields. Domination arises in facility location problems, where the number of facilities (e.g., hospitals, fire stations) is fixed and one attempts to minimize the distance that a person needs to travel to get to the closest facility. A similar problem occurs when the maximum distance to a facility is fixed and one attempts to minimize the number of facilities necessary so that every one is serviced. Concepts from domination set also appear in problems involving finding sets of representatives in monitoring communication or electrical networks, and in land surveyor must stand in order to take height measurements for an entire region. In this section, we introduce the concept of point set dominating set in vague graphs and semi complete vague graphs and obtain some interesting results for these new parameters.

Definition 3.1. Let $G=(V, E)$ be a vague graph. A set $D \subseteq V$ is said to be a point set dominating set or a psd-set of $G$ if for every $S \subseteq V-D$, there exists a node $d \in D$ such that the vague subgraph $\langle S \cup\{d\}\rangle$ induced by $S \cup\{d\}$ is a connected strong vague graph. The point set domination number $\gamma_{i p}(G)$ of $G$ is the minimum scalar cardinality of point set dominating sets.

Example 3.2. Consider vague graph $G$ in Figure 1.

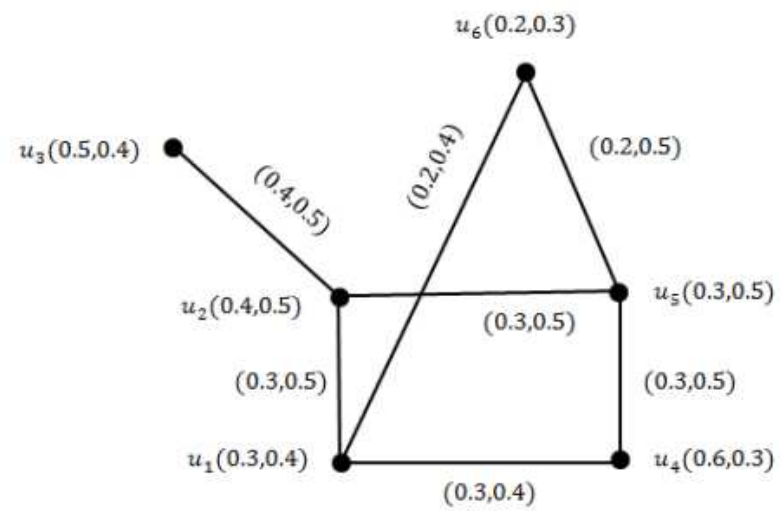

Figure 1: Vague graph $G$

By routine calculation we have: $\left|u_{1}\right|=0.45,\left|u_{2}\right|=0.45,\left|u_{3}\right|=0.55$, $\left|u_{4}\right|=0.65,\left|u_{5}\right|=0.4,\left|u_{6}\right|=0.45 . \gamma_{i p}$-set is $\left\{u_{3}, u_{4}, u_{6}\right\}$ and $\gamma_{i p}(G)=1.65$. 
H.Rashmanlou, A.Lakdashti and S.S.Sabet

Theorem 3.3. For any vague graph $G, \min \left\{\left|v_{i}\right|\right\} \leq \gamma_{i p}(G)<p$.

Proof: If $G$ is a complete vague graph, then $\gamma_{i p}(G)$-set contains only one vertex and we know that $\gamma_{i p}(G)$-set have at least one vertex. That is $\min \left\{\left|v_{i}\right|\right\} \leq \gamma_{i p}(G)$. Also, suppose that $S$ be the $p s d$-set and $V-S$ is not an empty set. Then $S$ contain at most $n-1$ vertices. That is the cardinality of $S$ is less than $P$. Therefore, $\gamma_{i p}(G)<P$. Hence, we get $\min \left\{\left|v_{i}\right|\right\} \leq \gamma_{i p}(G)<P$.

Remark 3.4. $(i) \gamma_{i p}\left(K_{n}\right)=\min \left\{\left|v_{i}\right|\right\}$, where $v_{i} \in V$.

(ii) $\gamma_{i p}\left(K_{v_{1 i}, v_{2 j}}\right)=\min \left\{\left|v_{1 i}\right|+\left|v_{2 j}\right|\right\}$, where $v_{i} \in V_{1}$ and $v_{j} \in V_{2}$.

Theorem 3.5. Every psd-set is a dominating set but the converse is not correct in general.

Proof: Let $S$ be $p s d$-set. By the definition, it is always a dominating set of $G$ but may not minimum. For the minimal dominating set $D$, there exist a vertex $d \in D$ such that $\langle S \cup\{d\}\rangle$ may not be connected and $S \subseteq V-D$. That is, converse need not be true.

Example 3.6. In Figure 1, $\gamma$-set $D=\left\{u_{1}, u_{2}\right\}, \gamma(G)=0.9$, and $\gamma_{i p}$-set is $\left\{u_{3}, u_{4}, u_{6}\right\}$. Here, $\gamma$-set is not $\gamma_{i p}(G)$-set. Since, for the set $S=\left\{u_{3}, u_{6}\right\} \subseteq V-D$, both $\left\langle S \cup\left\{u_{1}\right\}\right\rangle$ and $\left\langle S \cup\left\{u_{2}\right\}\right\rangle$ are disconnected. Also, $\gamma(G)<\gamma_{i p}(G)$.

Theorem 3.7. Let $G=(V, E)$ be a vague graph and $D$ is psd-set of $G$. Then $\langle V-D\rangle$ is a proper sub graph of a component $H$ of $G$.

Proof: Suppose there exist vertices $u$ and $v$ belonging to two different components of $G$. Since $D$ is a $p s d$-set of $G$, there exist $w \in D$ such that $\langle\{u, v, w\}\rangle$ is strongly connected vague graph which is contradiction to our assumption, i.e. $V-D \subseteq V(H)$, for some component $H$ of $G$. Also, $D \cap V(H) \neq \varnothing$ which implied $\langle V-D\rangle$ is a proper sub graph of $H$.

Theorem 3.8. Let $G=(V, E)$ be a vague graph. If $S$ is a psd -set and $u, v \in V-S$, then there are at most two strong arcs between $u$ and $v$.

Proof: Let $D=\{u, v\}, u$ and $v$ are not adjacent, and $S$ is the $p s d$-set. Then, there exist a vertex $x$ in $S$ such that the sub graph $\langle\{u, v, x\}\rangle$ is strongly connected. This implies there are at most two strong arcs between the vertices $u$ and $v$.

Theorem 3.9. Let $G=(V, E)$ be a strongly connected vague graph and $S$ is the dominating set of $G$ which is not psd-set in $G$. Then the minimal $\gamma$-set contains at least two vertices. 
A Study on Domination sets in Vague Graphs with Novel Applications

Proof: Since $S$ is not a $p s d$-set, there is a $D \subseteq V-S$ such that $\langle D \cup\{v\}\rangle$ is not connected for any $v \in S$. Hence, $\langle D\rangle$ is not connected and therefore it has at least two components. So, at least two vertices in $S$ are not dominated by a single vertex from $S$, which implies $\gamma$-set contains at least two vertices.

Theorem 3.10. Let $G=(V, E)$ be the purely semi complete vague graph and has a unique path between any pair of vertices in $G$. Then the intersection of all psd-sets in $G$ is a singleton set.

Proof: Since $G$ is purely semi complete vague graph, $G$ is a union of edge disjoint triangles having a common vertex, say $u$. It follows that any $p s d$-set contains the vertex $u$. Also, $\{u\}$ itself is $p s d$-set. Hence, their intersection is $\{u\}$.

Remark 3.11. The converse of the above theorem is not true i.e. if the intersection of all psd-sets in $G$ is a singleton set, then $G$ need not be a purely semi complete vague graph with unique path between any pair of vertices in $G$. Following example shows this subject.

Example 3.12. Consider purely semi-complete vague graph $G$ in Figure 2.

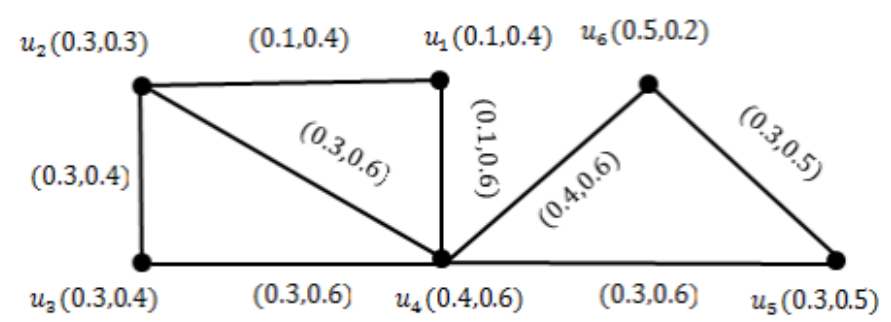

Figure 2: Purely semi-complete vague graph $G$

Here, all the $p s d$-sets include $u_{4}$. But there are two different shortest paths $\left\{u_{4}, u_{1}, u_{2}\right\}$ and $\left\{u_{4}, u_{3}, u_{2}\right\}$ between the vertices $u_{2}$ and $u_{4}$.

Theorem 3.13. Let $G=(V, E)$ be the purely semi complete vague graph with $n$ vertices and $S$ be the dominating set with number of vertices is greater than or equal to $n-2$. Then, $S$ is psd-set in $G$.

Proof: Let $D \subseteq V-S$ and assume that $S$ has $n-2$ vertices. Then either $D$ is singleton or contain two vertices. Let $D=\{v\}$. Since $S$ is dominating set, there is a $u \in S$ such that $v$ is adjacent to $u$. That is $\langle D \cup\{u\}\rangle$ is connected. Now, let $D=\left\{v_{1}, v_{2}\right\}$. If $v_{1}$ and $v_{2}$ are adjacent, then there is a $u \in S$ such that $\langle D \cup\{u\}\rangle$ is connected. Suppose that $v_{1}$ and $v_{2}$ are not adjacent. Then, by the nature of $S$, there is a $u \in S$ such that $\langle D \cup\{u\}\rangle$ is connected. Thus $S$ is psd-set in $G$. 
H.Rashmanlou, A.Lakdashti and S.S.Sabet

Theorem 3.14. Let $G=(V, E)$ be the purely semi complete vague graph and $S$ is a dominating set in $G$. Then, $S$ is psd-set if and only if for any independent vertex set $B \subseteq V-S$, there is a $v \in S$ such that every vertex of $B$ is strongly adjacent to $v$ in $G$.

Proof: Let $S$ be a $p s d$-set in $G, B$ be any independent vertex set, and $B \subseteq V-S$. Then, there is a $v \in S$ such that $\langle S \cup\{v\}\rangle$ is strongly connected. Since no two elements in $B$ are adjacent, by previous theorem there are two strong arcs between two vertices. Therefore, $G$ is purely semi complete vague graph and $S$ is a dominating set in $G$. Conversely, suppose $S$ is a dominating set in $G$ and for any independent vertex set $B \subseteq V-S$, there is a $v \in S$ such that every vertex of $B$ is strongly adjacent to $v$ in $G$. Let $D \subseteq V-S$ and $\langle D\rangle$ is connected. Then, $S$ is psd-set. Otherwise, let there are more than one components of $D$. Then $B=\left\{v_{1}, v_{2}, \cdots, v_{n}\right\}$ is an independent set in $G$. Now by hypothesis, since each $v_{i}$ is adjacent to $v$ in $G$, then there exist $v \in S$ such that $\langle B \cup\{v\}\rangle$ is connected. Therefore, $\langle D \cup\{v\}\rangle$ is connected. Hence, $S$ is psd -set in $G$.

Definition 3.15. Let $G=(V, E)$ be a vague graph. A set $D \subseteq V$ is said to be a set-domination set if for every set $T \subseteq V-D$, there exists a non-empty set $S \subseteq D$ such that the vague induced sub graph $\langle S \cup T\rangle$ is connected. The minimum cardinality taken over all set-domination sets is called set-domination number and denoted by $\gamma_{s}(G)$.

Example 3.16. In Example 3.2, it is easy to see that $\left\{u_{3}, u_{4}, u_{5}\right\}$ is a set-domination set.

Theorem 3.17. Let $G=(V, E)$ be a vague graph with effective edges. Then, $\gamma(G) \leq \gamma_{s}(G) \leq \gamma_{i p}(G)$.

Proof: Let $D$ be a point set dominating set of a vague graph. For every set $T \subseteq V-D$ there exist a non-empty singleton set $\{v\} \in S \subseteq D$ such that the sub graph $\langle S \cup T\rangle$ is connected. Thus, $D$ is a set-domination set. So, $\gamma_{s}(G) \leq \gamma_{i p}(G)$. Now, let $D$ be a set-domination set of a vague graph $G$. Then, for any set $\{x\}=T \subseteq V-D$, there exist a non-empty singleton set $\{v\} \in S \subseteq D$ such that the sub graph $\langle S \cup T\rangle$ is connected. Thus, $D$ is a domination set. So, $\gamma(G) \leq \gamma_{s}(G)$. Therefore, we have $\gamma(G) \leq \gamma_{s}(G) \leq \gamma_{i p}(G)$.

\section{Application of point set dominating sets in vague graphs}

In this section, we give some applications of point set dominating sets in the other sciences.

\subsection{Modeling social networks}

Point set dominating sets can be used in modeling social networks and studying the 
A Study on Domination sets in Vague Graphs with Novel Applications

dynamics of relations among numerous individuals in different domains. A social network is a social structure made of individuals (or groups of individuals), which are connected by one or more specific types of interdependency. The choice of initial sets of target individuals is an important problem in the theory of social networks. In the works of Kelleher and Cozzens, social networks are modeled in terms of graph theory and it was shown that some of these sets can be found by using the properties of dominating sets in graphs.

\subsection{Social groups}

It is observed that in social group some people can influence others and it can happen only when there is a strong relationship between them. Now, let us consider a vague graph of a social group as given in Figure 3.

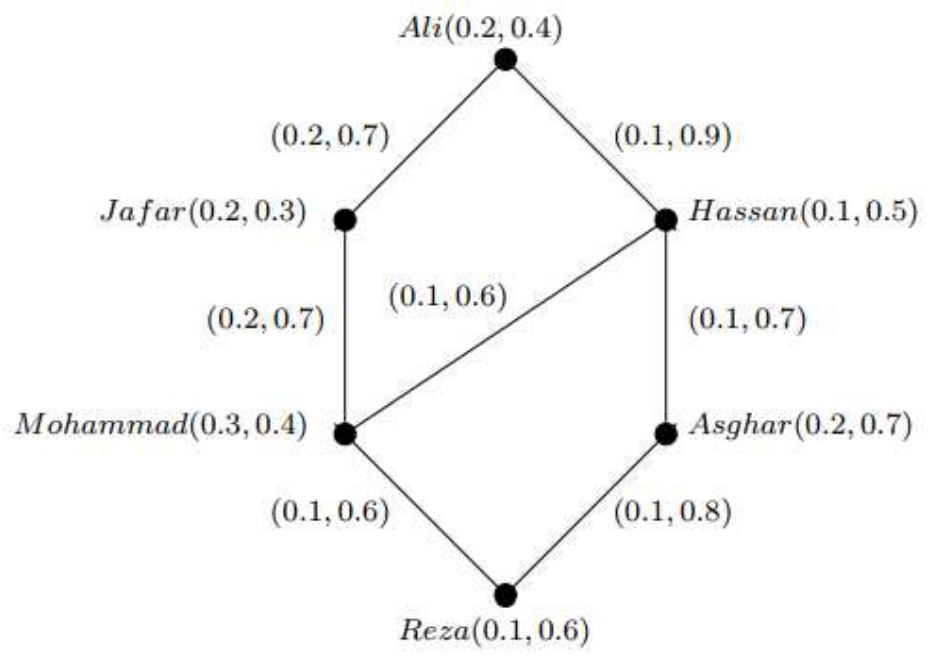

Figure 3: Vague graph $G$ of social relation

The nodes are depicting the degree of power of a person belongs to a set of social group. The degree of power of a person is defined in terms of its true membership and false membership. Degree of true membership can be interpreted as how much power a person possess and false membership can be interpreted as how much power a person losses. Ali has $20 \%$ power within the social group but he losses $40 \%$ power in the same group. In this example, \{Ali,Jafar\} , \{Asghar,Hassan\}, \{Jafar,Mohammad $\}$, and $\{$ Mohammad,Reza\} are strong edges. So, we can say there is a strong relation between Ali and Jafar. It is true for Asghar, Hassan and Jafar, Mohammad too. Hence, we see that Jafar dominates Ali,Hassan dominates Asghar, and Mohammad dominates Jafar. It is clear that \{Ali,Mohammad, Asghar\},

$$
\{\text { Ali,Mohammad,Asghar,Reza }\} \text {, and }\{\text { Ali,Mohammad,Reza,Hassan }\}
$$

are dominating sets. By routine calculation we have $\mid$ Ali $|=0.4$,$| Hassan \mid=0.3$, $\mid$ Reza $|=0.25$,$| Jafar \mid=0.45$, and $\mid$ Asghar $\mid=0.25$. 
H.Rashmanlou, A.Lakdashti and S.S.Sabet

$\gamma_{i p}$-set is $\{$ Jafar, Ali,Hassan $\}$. The edges of a vague graph show the influence of one person to another person. The degree of true membership and false membership of edges can be interpreted as the percentage of true membership and false membership influence. For example, Hassan follows 10\% Mohammad's suggestions but he/she does not follows $60 \%$ his/her suggestions. Therefore, domination can help us to find people who have strong relation in a social group.

\subsection{Social network theory}

The online social network has been developed significantly in the recent years as a medium of communication, sharing the information and spreading the influence. Most of the current research has been carried out on understanding the property of how the online social network has been utilized in disseminating the information and ideas. The problem of utilizing the online social network for solving the social problems in the physical world such as drinking, smoking, and drug problems are all explored well. The dominating set plays a vital role in analyzing the effect on a real online social network data set through simulation. The dominating set concept can be applied to the social network graph to determine the amount of positive influence that is possessed by an individual as well as its impact to their related neighbor. This dominating positive degree threshold can spread the positive educational influence throughout the entire community in the social network.

In a social setup people may have both positive as well as negative impact on each other and persons can play different roles as they are affected by their peers. The positive or negative influence in social issues can move in two directions. That is, a positive individual can convert into a negative individual and can move back and forth between these two states for multiple times. A vague undirected graph $G=(V, E, C)$ is used to denote the online social network, because friendship in an online social network are usually bidirectional as shown in below.

Online social network can be represented as a graph of relationship with individuals representing the nodes of a graph $(V)$, the social interactions as edges $(E)$ and $C$ is the compartment vector that saves the compartment of each node. The compartment part of a node decides whether the social issue(s) of an individual has positive or negative impact on its neighbors.

\section{Conclusion}

It is well known that graphs are among the most ubiquitous models of both natural and human-made structures. They can be used to model many types of relations and process dynamics in computer science, physical, biological, and social systems. In general graph theory has a wide range of applications in diverse fields. In this paper, we studied some results on point set dominating set and its number of vague graphs and semi complete vague graph. Also, we introduced the bounds with other domination parameters of vague graphs.

\section{REFERENCES}

1. M.Akram, N.Gani and A.B.Saeid, Vague hypergraphs, Journal of Intelligent and Fuzzy Systems, 26 (2014) 647-653.

2. R.A.Borzooei and H.Rashmanlou, Domination in vague graphs and its applications, 
A Study on Domination sets in Vague Graphs with Novel Applications Journal of Intelligent and Fuzzy Systems, 29 (2015) 1933-1940.

3. W.L.Gau and D.J.Buehrer, Vague sets, IEEE Transactions on Systems, Man and Cybernetics, 23 (2) (1993) 610-614.

4. A.Kauffman, Introduction a la theorie des sous-emsembles 503 ous, Masson et Cie 1 (1973).

5. A.Nagoorgani, S.Y.Mohamed and R.J.Hussain, Point set domination of intuitionistic fuzzy graphs, International Journal of Fuzzy Mathematical Archive, 7 (1) (2015) 43-49.

6. R.Parvathi and G.Thamizhendhi, Domination in intuitionistic fuzzy graphs, Fourteenth Int.Conf.on IFSs, Sofa 16 (2) (2010), 39-49.

7. N.Ramakrishna, Vague graphs, International Journal of Computational Cognition, 7 (2009), 51-58.

8. H.Rashmanlou, S.Samanta, M.Pal and R.A.Borzooei, A study on bipolar fuzzy graphs, Journal of Intelligent and Fuzzy Systems, 28 (2015) 571-580.

9. H.Rashmanlou, S.Samanta, M.Pal and R.A.Borzooei, Bipolar fuzzy graphs with categorical properties, International Journal of Computational Intelligent Systems, 8 (5) (2015) 808-818.

10. H.Rashmanlou, S.Samanta, M.Pal and R.A.Borzooei, Product of bipolar fuzzy graphs and their degree, International Journal of General Systems, doi.org/10.1080/03081079.2015.1072521.

11. H.Rashmanlou and R.A.Borzooei, Product vague graphs and its applications, Journal of Intelligent and Fuzzy Systems, 30 (2016) 371-382.

12. H.Rashmanlou, R.A.Borzooei, Sankar Sahoo, M. Pal,On searching vague shortest path in a network, Journal of Multiple-Valued Logic and Soft Computing, 29 (2017) 355-372.

13. H.Rashmanlou, S.Samanta, M.Pal and R.A.Borzooei, Properties of interval valued intuitionistic (S-T) fuzzy graphs, Pacific Science review, doi.org/10.1016/j.psra.1016.06.003.

14. H.Rashmanlou, S.Samanta, M.Pal, R.A.Borzooei, Intuitionistic fuzzy graphs with categorical properties, Fuzzy Information and Engineering, 7 (2015) 317-334.

15. H.Rashmanlou and M.Pal, Some properties of highly irregular interval-valued fuzzy graphs, World Applied Sciences Journal, 27 (2013) 1756-1773.

16. H.Rashmanlou and M.Pal, Balanced interval-valued fuzzy graph, Journal of Physical Sciences, 17 (2013) 43-57.

17. A.Somasundaram and S.Somasundaram, Domination in fuzzy graphs-I, Pattern Recognition Letters, 19 (1998) 787-791.

18. D.H.Sanford, Vague numbers, Acta Analytica, 17 (2) (2002) 63-73.

19. L.A.Zadeh, Fuzzy sets, Information and Control, 8 (1965) 338-353.

20. S.Samanta and M.Pal, Fuzzy $k$-Competition Graphs and p-Competition Fuzzy Graphs, Fuzzy Inf. Eng., 5(2) (2013) 191-204.

21. S. Samanta, A. Pal and M. Pal, New concepts of fuzzy planar graphs, International Journal of Advanced Research in Artificial Intelligence, 3(1) (2014) 52-59.

22. S.Samanta, M.Pal and A.Pal, Some more results on fuzzy k-competition graphs, International Journal of Advanced Research in Artificial Intelligence, 3(1) (2014) 60-67. 
H.Rashmanlou, A.Lakdashti and S.S.Sabet

23. S.Samanta and M.Pal, Fuzzy Planar Graphs, IEEE Transaction on Fuzzy System, 23(6) (2015) 1936 - 1942.

24. S.Samanta, M.Akram and M.Pal, m-step fuzzy compitition graphs, Journal of Applied Mathematics and Computing, 47 (2015) 461-472.

25. T. Pramanik, S. Samanta and M. Pal, Interval-valued fuzzy planar graphs, Int. J. Mach. Learn. \& Cyber., 7(4) (2016) 653-664.

26. R. A. Borzooei, H. Rashmanlou, S. Samanta and M. Pal, A Study on Fuzzy Labeling Graphs, Journal of Intelligent and Fuzzy Systems, 30 (2016) 3349-3355.

27. R. A. Borzooei, H. Rashmanlou and M. Pal, Regularity of vague graphs, Journal of Intelligent and Fuzzy Systems, 30 (2016) 3681-3689.

28. S.Samanta, M.Pal, R.A.Borzooei and H.Rashmanlou, Vague graphs and strengths, Journal of Intelligent and Fuzzy Systems, 30 (2016) 3675-3680.

29. G. Ghorai and M. Pal, Faces and dual of m-polar fuzzy planar graphs, Journal of Intelligent and Fuzzy System, 31 (2016) 2041-2049.

30. S.Samanta, T.Pramanik and M.Pal, Fuzzy colouring of fuzzy graphs, Afrika Matematika, 27(1-2) (2016) 37-50.

31. G.Ghorai and M.Pal, A study on m-polar fuzzy planar graphs, Int. J. Computing Science and Mathematics, 7(3) (2016) 283-292.

32. G.Ghorai and M.Pal, Some isomorphic properties of m-polar fuzzy graphs with applications, SpringerPlus, 5:1-21 (2016).

33. T.Pramanik, M.Pal and S.Mondal, Interval-valued fuzzy threshold graph, Pacific Science Review A: Natural Science and Engineering, 18 (2016) 66-71.

34. S.Samanta, H.Rashmanlou, M.Pal and R.A.Borzooei, A study on vague graphs, SpringerPlus, (2016) 5:1234.

35. S.Sahoo and M.Pal, Intuitionistic fuzzy competition graphs, J. Appl. Math. Comput., 52(1) (2016) 37-57.

36. G.Ghorai and M.Pal, Regular product vague graphs and product vague line graphs, Cogent Mathematics, 3(1) (2016) Article. 1213214.

37. T.Pramanik, S.Samanta, B.Sarkar and M.Pal, Fuzzy $\varphi$-tolerance competition graphs, Soft Computing, 21 (2017) 3723-3734.

38. S. Sahoo and M. Pal, Intuitionistic fuzzy tolerance graphs with application, J. Appl. Math. Comput., 55 (2017) 495-511.

39. M.Hota, M.Pal and T.K.Pal, An efficient algorithm for finding a maximum weight k-independent set on trapezoid graphs, Computational Optimization and Applications, 18 (1) (2001) 49-62.

40. M.Hota, M.Pal and T.K.Pal, An efficient algorithm to generate all maximal independent sets on trapezoid graphs, International Journal of Computer Mathematics, 70 (4) (1999) 587-599.

41. M.Pal and G.P.Bhattacharjee, A sequential algorithm for finding a maximum weight $\mathrm{k}$-independent set on interval graphs, International Journal of Computer Mathematics, 60 (3-4) (1996) 205-214.

42. S.Mondal, M.Pal and T.K.Pal, An optimal algorithm for solving all-pairs shortest paths on trapezoid graphs, International Journal of Computational Engineering Science, 3 (2) (2002) 103-116.

43. M.Pal, Intersection graphs: An introduction, Annals of Pure and Applied Mathematics, 4 (1) (2013) 41-93. 